\title{
Tamizaje y prevención de preeclampsia guiado por Doppler de arterias uterinas: revisión sistemática de la literatura
}

\author{
Nicolás Sáez O. ${ }^{1}$, Jorge Carvajal C. ${ }^{2}, P h D$. \\ 1 Programa de Obstetricia y Ginecología, ${ }^{2}$ Unidad de Medicina Materno Fetal, División de Obstetricia y Ginecología, \\ Facultad de Medicina, Pontificia Universidad Católica de Chile.
}

\section{RESUMEN}

Antecedentes: La preeclampsia (PE) es una enfermedad grave asociada al embarazo con una prevalencia de $\sim 3 \%$. La identificación de las mujeres en riesgo podría mejorar el resultado materno y perinatal. La estimación de riesgo individual de PE permitiría ofrecer un tratamiento preventivo para ésta [ej: aspirina (AAS)]. Se ha descrito el Doppler de arterias uterinas (DAUt) en primer y segundo trimestre como un método de tamizaje de PE. Objetivo: Recopilar la evidencia disponible sobre el uso del DAUt para predecir PE y los potenciales beneficios de la profilaxis con AAS. Método: Se realizó una búsqueda en Pubmed, revisándose 32 trabajos originales, 5 revisiones y 1 meta-análisis. La calidad de los estudios se analizó según guías de análisis crítico de publicaciones científicas y de estudios que evalúan exámenes diagnósticos. Resultados: Luego de analizar todos los trabajos y resultados, se encuentra que la mejor capacidad diagnóstica del DAUt es la medición del índice de pulsatilidad promedio por vía transvaginal entre las 20 y 24 semanas en población de alto riesgo para predecir PE de inicio precoz. Utilizando el p95 como valor de corte se logra sensibilidad: $85 \%$, especificidad: $95 \%$, valor predictivo positivo: $18 \%$. AAS se ha mostrado efectiva en la prevención de PE en población de riesgo, con disminuciones marginales $(\sim 17 \%)$ en su incidencia y sin disminuir la morbimortalidad materna ni perinatal. Conclusión: El DAUt es un método útil para el tamizaje de PE precoz en pacientes embarazadas seleccionadas por factores de riesgo, pero se necesitan más estudios evaluando la utilidad del AAS como profilaxis de PE en dicha población.

\section{PALABRAS CLAVE: Preeclampsia, doppler de arterias uterinas, tamizaje, prevención}

\section{SUMMARY}

Background: Preeclampsia (PE) is a pregnancy-related severe disease with a prevalence of $\sim 3 \%$. Identification of women at risk may improve maternal and perinatal outcome. Estimate of PE individual risk would offer preventive treatment [ie: Aspirin (ASA)]. Uterine Artery Doppler (UAD) has been used in first and second trimester as a PE screening test. Aims: To gather the available evidence on PE prediction with UAD and the potential benefits of ASA use in these women. Methods: We searched on Pubmed, reviewing 32 original papers, 5 reviews and 1 meta-analysis. The study quality was analyzed with scientific literature review guidelines and diagnostic test analysis guidelines. Results: Literature analysis showed that UAD's best diagnostic capacity for early onset PE is through trans-vaginal mean pulsatility index measurement between 20th and 24th week in high risk population. Eighty five percent sensibility ( $85 \%$ specificity), with $18 \%$ PPV is achieved using p95 as a cut value. ASA is effective in preventing PE in high risk population, with $17 \%$ 
decrease in incidence but with no effect on maternal or perinatal morbid-mortality. Conclusion: UAD is useful in early onset PE screening in pregnant population selected by risk factors. Further studies are needed to direct ASA use as PE prophylaxis in this population.

\section{KEY WORDS: Preeclampsia, uterine artery doppler, screening, prevention}

\section{INTRODUCCIÓN}

La preeclampsia (PE) es una enfermedad grave asociada al embarazo con una prevalencia aproximada de $3 \%$ (1). La presencia de PE confiere riesgos perinatales de morbimortalidad. Entre las complicaciones maternas existe un aumento del riesgo de infarto miocárdico, enfermedad cerebro-vascular (ECV) o diabetes mellitus (DM), 2 a 8 veces en las dos décadas siguientes al diagnóstico (2), mientras en el neonato hay un riesgo de restricción del crecimiento fetal (RCF), hipertensión crónica, ECV, DM o enfermedad renal en la adultez de 2 a 8 veces $(3,4)$. No existen métodos para predecir la progresión de la PE y no existe cura para ésta, a excepción del parto.

La identificación de las mujeres en riesgo, el diagnóstico precoz y el acceso oportuno al manejo apropiado (corticoides antenatales, tratamiento de la hipertensión severa, interrupción del embarazo) pueden mejorar el resultado materno y, probablemente el perinatal (5). La estimación de riesgo individual de PE permitiría, además, ofrecer un eventual tratamiento preventivo, como es el uso de ácido acetilsalicílico (AAS).

Un buen examen de tamizaje para PE debiera ser simple, rápido, barato y fácil de realizar/reproducir sin exponer a la paciente a riesgo o incomodidad física (6). La tecnología debiera estar disponible y los resultados debieran ser confiables con un likelihood ratio positivo (LR+) $>10$ y un likelihood ratio negativo (LR-) $<0,1$. Idealmente debiera permitir el acceso a la intervención preventiva para el desarrollo de la enfermedad o a lo menos derivar en un mejor resultado materno/fetal (7).

Se han descrito numerosos métodos de tamizaje, entre los que destacan los factores epidemiológicos, el Doppler de arterias uterinas (DAUt) en primer y segundo trimestre, y diversos marcadores séricos como: hCG libre, PAPP-A, VEGF, PIGF, ADAM-12, sFlt-1, inhibina-A, activina-A (8) (Tabla I).

El tamizaje de PE por historia materna detecta sólo el $30 \%$ de las PE (9) y es ineficaz en pacientes nulíparas sanas. Sin embargo, la nuliparidad es uno de los factores más importantes en el desarrollo de PE (Tabla I) $(10,11)$.

La literatura disponible para tamizaje de PE con
Tabla I

\section{FACTORES DE RIESGO ASOCIADOS AL} DESARROLLO DE PREECLAMPSIA

\begin{tabular}{ll}
\hline Factor de riesgo & RR ajustado (IC95\%) \\
\hline Nuliparidad & $3,1(1,55-6,17)$ \\
PE en embarazo previo & $7,19(5,85-8,83)$ \\
Edad extrema & \\
$\quad>40$ años & $1,96(1,34-2,87)$ \\
$\quad<18$ años & $2,98(0,39-22,76)$ \\
Historia familiar de preeclampsia & $2,9(1,7-4,93)$ \\
Hipertensión arterial crónica & $3,6(2,0-6,6)$ \\
Enfermedad renal & $2,94($ no disponible) \\
Síndrome de anticuerpos & $9,72(4,34-21,75)$ \\
antifosfolípidos & \\
Enfermedad del tejido conectivo & $6,9(1,1-42,3)$ \\
o vascular & \\
Diabetes pregestacional insulina & $3,56(2,54-4,99)$ \\
requirente & \\
Embarazo gemelar & $2,93(2,04-4,21)$ \\
Indice masa corporal elevado & $2,1(1,0-4,6)$ \\
Período intergenésico & $1,12(1,11-1,13)$ \\
prolongado ( $\geq 10$ años) & \\
Fumadora & $0,66(0,61-0,71)$ \\
$\quad$-social &
\end{tabular}

DAUt muestra resultados variables, dando cabida a dudas de cuando aplicar el tamizaje y a que pacientes en particular.

Se ha estudiado la utilidad del tamizaje de PE sumando el DAUt y los marcadores séricos. Los resultados son contradictorios, los marcadores son de disponibilidad limitada en la población chilena y no existen guías clínicas que nos permitan orientar su uso $(12,13,14)$. Desde la perspectiva de la prevención de PE, la estrategia más usada es la administración profiláctica de AAS en dosis baja. Se ha estudiado su uso en población de alto o bajo riesgo e incluso la asociación al resultado del DAUt.

El objetivo de esta revisión sistemática es recopilar la evidencia disponible sobre el uso del DAUt para predecir PE, entregar directrices en cuanto a su utilidad, el momento oportuno de aplicación, selección de pacientes y los potenciales beneficios de la profilaxis 
con AAS. No se encuentra dentro de los objetivos de este estudio el análisis de la utilidad de los marcadores séricos maternos en el tamizaje de PE.

\section{MÉTODO}

Se realizó una búsqueda en Pubmed con los criterios de búsqueda "Screening" AND "Pre-eclampsia" AND "uterine artery doppler". Se utilizaron como límites: sexo femenino, idiomas inglés y español y que la publicación fuera de los últimos 10 años. Se obtuvieron 70 resultados, de los cuales se consideraron 45 relevantes por título y resumen. Se logró obtener el texto completo de 33 artículos los cuales fueron revisados. Se obtuvo el texto completo de citas frecuentes y/o relevantes que no fueron encontradas en nuestra búsqueda primaria y, además, se buscaron estudios en curso sobre el tópico registrados en la International Clinical Trials Registry Platform (ICTRP) de la OMS (http://www.who.int/ictrp), encontrándose 3 registros que evalúan uso de DAUt y administración de AAS en bajas dosis o heparina no fraccionada como prevención de PE.

Se definió PE como hipertensión arterial ( $\geq 140 / 90$ ), con proteinuria (proteínas totales $\geq 300$ $\mathrm{mg} / 24 \mathrm{~h}$ o dipstick ++ o más) después de las 20 semanas de gestación (15). La calidad de los estudios se analizó según guías de análisis crítico de publicaciones científicas (16) y de estudios que evalúan exámenes diagnósticos (17).

\section{RESULTADOS}

Se analizó un total 32 trabajos originales, y 5 revisiones. Además se encontró un meta-análisis que evalúa la utilidad del AAS en pacientes con DAUt alterado. De los trabajos originales son: 25 prospectivos, 3 caso-control y 4 estudios clínicos aleatorizados y controlados, que evalúan la utilidad del AAS en bajas dosis en distintos momentos del embarazo.

Dentro de los estudios prospectivos se distinguen cuatro grupos de diseño: los que evalúan la utilidad del DAUt (con o sin factores maternos) en el primer trimestre, los que evalúan su utilidad en el segundo trimestre, los que agregan marcadores séricos al DAUt en primer trimestre y los que agregan marcadores séricos al DAUt en segundo trimestre.

\section{Doppler de arterias uterinas (DAUt)}

El DAUt es una técnica descrita en 1983 (18), siendo propuesta como prueba para predecir riesgo de PE, RCF, y otros resultados perinatales adversos. El principio fisiopatológico de su uso se basa en la representación flujométrica en las arterias uterinas de una resistencia aumentada, dada por una invasión trofoblástica defectuosa y el fallo en la conversión de las arteriolas uterinas de alta resistencia a canales venosos de baja resistencia (19). Se postula que la PE de inicio precoz (antes de las 34 semanas) se basa en esta fisiopatología (invasión trofoblástica defectuosa) y se asocia más a RCF, constituyendo un cuadro severo, mientras que la PE que se manifiesta en embarazos de término no compartiría este mecanismo $(20,38)$.

La técnica puede ser por vía transvaginal (TV) o abdominal. La mayoría de los estudios de primer trimestre son por vía abdominal, aprovechando el tamizaje para aneuploidías con medición de LCN (45 a $84 \mathrm{~mm}$ ) y traslucidez de nuca que se realiza entre las 11 y 14 semanas de gestación. En el $2^{\circ}$ trimestre suele utilizarse la técnica por vía TV (descrita en http://www.fetalmedicine.com), aprovechando el tamizaje para parto prematuro entre las 20 y 24 semanas $(9,12,13,16,21,26-30,36-38,42)$. Los grupos que no realizan tamizaje para parto prematuro en población general, utilizan la vía abdominal aprovechando el tamizaje de malformaciones en el mismo período del embarazo.

En la década pasada, era más común el uso del índice de resistencia (IR) o consignar la presencia de "notch" o escotadura protodiastólica, con alta subjetividad y variación interobservador. Actualmente, se utiliza el IP promedio entre ambas arterias uterinas con el valor p95 como punto de corte de normalidad/anormalidad (21). Se recomienda el uso de tablas de valores normales de IP según edad gestacional, pues es sabido que a medida que progresa el embarazo el IP va disminuyendo, como manifestación de la disminución de la resistencia vascular dado por el proceso de placentación fisiológico. Se recomienda utilizar IP p95 en primer trimestre (valor $=2,43$ ) y en segundo trimestre (valor=1,54) basado en datos obtenidos en población chilena y comparable con literatura internacional (22).

\section{DAUt en primer trimestre vs segundo trimestre}

Encontramos nueve estudios que evalúan la efectividad del DAUt (con o sin marcadores séricos) como predictor de PE en primer trimestre (11-14 semanas) (21-29). El DAUt como método de tamizaje en población no seleccionada y utilizando como valor de corte el percentil 95 (p95) del índice de pulsatilidad (IP), tiene sensibilidad (S) 25\% para PE en globo y $\sim 60 \%$ para $P E$ precoz, con especificidad $(E)$ $95 \%$, valor predictivo positivo (VPP) que no supera el $10 \%$ y valor predictivo negativo (VPN) cercano a $100 \%$. Estos resultados han sido corroborados en 
un estudio en población chilena (22) y por múltiples estudios internacionales $(23,24,25)$. Al adicionar los factores epidemiológicos (26) y presión arterial $(27,28,29)$ al control, se demuestran resultados aún mejores para PE precoz, con S: 89\%, E: 90\%, LR+: $\sim 8$ y LR-: $~ 0,2$. El mejor valor del DAUt para predecir PE en primer trimestre es el IP menor medido por vía abdominal (27). Los resultados se resumen en la Tabla II.

Encontramos diez estudios que evalúan la efectividad del DAUt (con o sin marcadores séricos) como predictor de PE en segundo trimestre (20-24 semanas) (22,30-38). Como método de tamizaje en población no seleccionada y utilizando como valor de corte el p95 del IP promedio entre ambas arterias uterinas (valor: 1,6) se obtiene para PE precoz una S: $80 \%$, E: 95\% y LR+: 15,8 (30). Estos valores han sido corroborados en población chilena (22) y son mejores que los de primer trimestre (31-33).

Sólo un estudio evalúa la diferencia entre población de alto y bajo riesgo. En población de alto riesgo, el rendimiento del DAUt es mayor tanto para predecir PE como RCF y desprendimiento de placenta (34), y dado su alto VPN (cercano al $100 \%$ ), el tener un DAUt normal disminuye el riesgo de PE al mismo que de la población general (35). El mejor valor de DAUt para predecir PE en segundo trimestre es el IP promedio medido por vía transvaginal (36).

En resumen, la mejor capacidad diagnóstica del DAUt es la medición del IP promedio por vía transvaginal entre las 20 y 24 semanas en población de alto riesgo (incluyendo factores epidemiológicos dentro del tamizaje) para predecir PE de inicio pre$\operatorname{coz}(37,38)$. En dichas circunstancias y utilizando el p95 como valor de corte se logra una S:85\% y E: 95\%, con VPP: 18\%; VPN: 100\% y LR+: 15 (ver ejemplo en Figura 1). Los resultados se resumen en la Tabla III.

\section{Utilidad de ácido acetilsalicílico (AAS) en bajas dosis para prevención}

La utilidad del AAS en bajas dosis para prevenir PE se ha estudiado en población general (sin uso de DAUt) y ha sido evaluada en meta-análisis. Duley y cols (39), evaluaron 59 estudios con un total de 37.560 embarazos, reportaron que el uso profiláctico de AAS en bajas dosis se asocia a una reducción moderada pero significativa $(17 \%$ [RR: 0,83; IC95\%: 0,77-0,89] NNT: 72) en la incidencia de PE en mujeres que comienzan el tratamiento antes de las 20 semanas. Sin embargo, los estudios varían en criterios de inclusión, nivel de riesgo de cada embarazo, dosis de aspirina y edad gestacional al ingreso. Además, se sugiere que la intervención es más efectiva en pacientes de alto riesgo (NNT: 19) al compararlas con pacientes de bajo riesgo (NNT: 119).

Se encontraron 4 estudios aleatorizados que evalúan la utilidad de la aspirina en bajas dosis para prevenir $\mathrm{PE}$ luego de seleccionar poblaciones de alto riesgo con DAUt:

Estudio multicentrico aleatorizado (40), DAUt a 1481 mujeres con embarazo de bajo riesgo, entre las 20 y 24 semanas. Administraron AAS $(100$ mg/ día) versus placebo a las pacientes con DAUt alterado hasta las 35 semanas, no encontrando efecto en la incidencia de PE o SHE al administrar AAS (RR:1,99 [IC95\%: 0,97-4,09]). Concluyen que no se justifica el tamizaje con DAUt en población de bajo riesgo, incluso si se administra AAS a los alterados y se les sigue de cerca en el control prenatal.

Tabla II

ESTUDIOS QUE REPORTAN S, E, VPP, VPN, LR+ Y LR- PARA LA PREDICCIÓN DE PREECLAMPSIA Y PREECLAMPSIA PRECOZ EN PRIMER TRIMESTRE EN PACIENTES CON EMBARAZO DE BAJO RIESGO

\begin{tabular}{|c|c|c|c|c|c|c|c|c|c|c|c|c|c|}
\hline \multirow[b]{2}{*}{ Referencia } & \multirow[b]{2}{*}{$\mathrm{n}$} & \multicolumn{2}{|c|}{ S (\%) } & \multicolumn{2}{|c|}{$E(\%)$} & \multicolumn{2}{|c|}{ VPP (\%) } & \multicolumn{2}{|c|}{ VPN (\%) } & \multirow{2}{*}{$\begin{array}{r}\text { LR+ } \\
\text { PE }\end{array}$} & \multicolumn{3}{|c|}{ LR- } \\
\hline & & $\mathrm{PE}$ & PE pr & $\mathrm{PE}$ & PE pr & PE & PE pr & PE & PE pr & & PE pr & PE & PE pr \\
\hline Martin $2001^{21}$ & 3045 & 27 & 60 & 95 & 95 & 11 & 3,9 & 98,4 & 99,9 & 5,4 & 12 & 0,76 & 0,42 \\
\hline Parra $2005^{22}$ & 743 & 25 & 66 & 95 & 95 & 10 & 6,8 & 98 & 99 & 5,3 & 13,5 & 0,79 & 0,35 \\
\hline Gomez $2005^{23}$ & 999 & 24 & $\mathrm{n} / \mathrm{d}$ & 95 & $\mathrm{n} / \mathrm{d}$ & 11 & $\mathrm{n} / \mathrm{d}$ & 98 & $\mathrm{n} / \mathrm{d}$ & 4,8 & $\mathrm{n} / \mathrm{d}$ & 0,8 & $\mathrm{n} / \mathrm{d}$ \\
\hline Pilalis $2007^{24}$ & 1123 & 21 & 33 & 95 & 95 & 5,4 & 3,5 & 98,9 & 99,6 & 4,2 & 6,6 & 0,83 & 0,7 \\
\hline Plasencia $2007^{26}$ & 3107 & 61 & 82 & 90 & 90 & 10 & $n / d$ & 99,2 & $\mathrm{n} / \mathrm{d}$ & 6,1 & 8,2 & 0,43 & 0,2 \\
\hline Poon $2009^{27}$ & 8366 & 57 & 89 & 90 & 90 & 10,3 & 3,8 & 99 & 99,9 & 5,7 & 8,9 & 0,47 & 0,12 \\
\hline Audibert $2010^{25}$ & 893 & 35 & 50 & 90 & 90 & 14,1 & 5,3 & 96,7 & 99,5 & 3,5 & 5 & 0,72 & 0,55 \\
\hline
\end{tabular}

$\mathrm{n} / \mathrm{d}=$ no disponible. S: sensibilidad. E: especificidad. VPP: valor predictivo positivo. VPN: valor predictivo negativo. LR: likelihood ratio. PE: preeclampsia. PE pr: Preeclampsia precoz. 


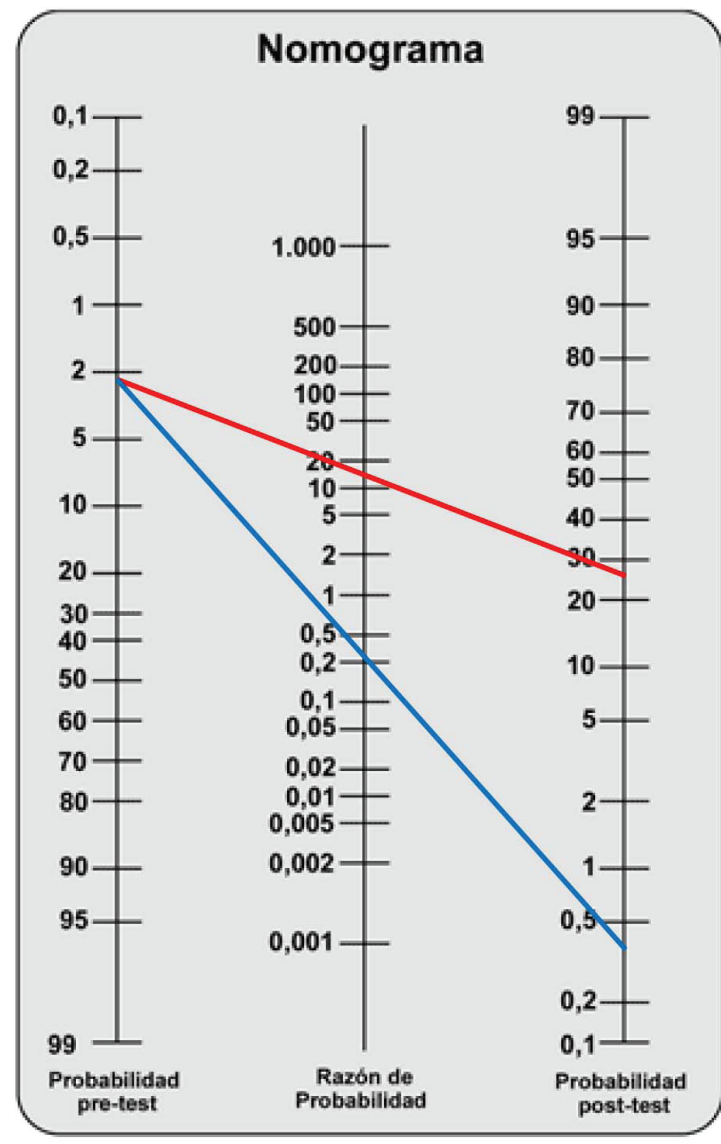

Nomograma reproducido con autorización de Edgardo Sandoya. Rev Urug Cardiol 2009;24:2840.

Caso: Paciente con alta probabilidad de desarrollar preeclampsia precoz por antecedentes clínicos (probabilidad pretest $2,5 \%$ ). Considerando un DAUt $2^{\circ}$ trimestre alterado con $L R+=15$, la probabilidad postest de desarrollar PE precoz es $\sim 25 \%$ (línea roja). Si DAUt $2^{\circ}$ trimestre es normal con LR$=0,2$; la probabilidad postest de desarrollar PE precoz es $\sim 0,4 \%$ (línea azul).

\section{LR = Razón de Probabilidad}

Figura 1. A modo de ejemplo, considerar probabilidad pretest $2,5 \%$ para preeclampsia precoz en población de alto riesgo, donde el DAUt demuestra su mayor utilidad.
Estudio multicéntrico aleatorizado y controlado (41), donde evaluaron 239 pacientes nulíparas con DAUt anormal en segundo trimestre y administraron AAS (100 mg/día) o placebo a las mujeres con DAUt alterado, hasta las 34 semanas. No encontraron diferencias significativas en la incidencia de PE (RR 1,55 [IC95\%: 0,7-3,3]).

Estudio aleatorizado y controlado (42), donde asignaron 560 mujeres con DAUt anormal a las 23 semanas a recibir AAS (150 mg/día) o placebo hasta las 36 semanas de embarazo. No encontraron diferencias significativas entre el grupo tratamiento y grupo placebo en incidencia de PE $(18,7 \%$ vs $17,8 \%$ ) o PE precoz ( $7,6 \%$ vs $6,2 \%)$. El uso de AAS no alteró ningún resultado perinatal adverso de forma significativa. Concluyeron que en mujeres con DAUt alterado no se justifica la administración de aspirina dado que no previene el desarrollo de PE ni mejora el resultado perinatal.

Estudio aleatorizado y controlado (43), donde evaluaron 139 mujeres en riesgo de PE o RCIU con DAUt alterado entre las 14 y 16 semanas. Se aleatorizó a AAS (75 mg/día) o placebo; $62 \%$ de las pacientes que recibieron placebo desarrollaron $\mathrm{PE}$, en comparación con un $35 \%$ de las pacientes con AAS $(p<0,001) .23 \%$ de las pacientes que recibieron placebo desarrollaron PE severa, en comparación con un $8 \%$ de las pacientes con AAS $(p=0,215)$. No hubo diferencias en el resultado materno o neonatal entre los dos grupos. Concluyeron que el AAS en bajas dosis puede reducir la incidencia de PE en mujeres con embarazo de alto riesgo (dado por factores epidemiológicos) y DAUt alterado, sin embargo, hay que ser cauteloso con los resultados demostrados dada la alta incidencia de PE en el grupo estudiado, sobre todo considerando la mala $S$ del DAUt en primer trimestre, que no se condice con ninguna publicación de las encontradas.

Recientemente, Fox y cols (44), publicaron un meta-análisis que resume 8 estudios con DAUt alterado y aleatorización a AAS en bajas dosis o placebo. Realizan un análisis sobre la utilidad de administrar AAS a distintas edades gestacionales y demuestran una disminución de un $27 \%$ (RR: 0,73; IC95\%: 0,58-0,92) de PE en globo, sin haber efecto si las pacientes se reclutan después de las 16 semanas de gestación. Se recomienda ser cuidadoso con la interpretación de este meta-análisis dada la exclusión de dos trabajos que no favorecen el uso de AAS $(40,41)$ y la inclusión de un trabajo con incidencias de PE sospechosamente elevadas en grupo placebo y tratamiento (43). 
Tabla III

ESTUDIOS QUE REPORTAN S, E, VPP, VPN, LR+ Y LR- PARA LA PREDICCIÓN DE PREECLAMPSIA Y PREECLAMPSIA PRECOZ EN SEGUNDO TRIMESTRE

\begin{tabular}{|c|c|c|c|c|c|c|c|c|c|c|c|c|c|}
\hline \multirow[b]{2}{*}{ Referencia } & \multirow[b]{2}{*}{$\mathrm{n}$} & \multicolumn{2}{|c|}{ S (\%) } & \multicolumn{2}{|l|}{$E(\%)$} & \multicolumn{2}{|c|}{ VPP (\%) } & \multicolumn{2}{|c|}{ VPN (\%) } & \multirow{2}{*}{$\begin{array}{r}\text { LR+ } \\
\text { PE }\end{array}$} & \multicolumn{3}{|c|}{ LR- } \\
\hline & & PE & PE pr & $\mathrm{PE}$ & PE pr & PE & PE pr & PE & PE pr & & PE pr & PE & PE pr \\
\hline $\begin{array}{l}\text { Papageorghiou } \\
2001^{30}\end{array}$ & 7851 & 24 & 80 & 95 & 95 & 4,2 & 1 & 99,3 & 100 & 4,9 & 15,8 & 0,8 & 0,21 \\
\hline Aquilina $2001^{31}$ & 640 & 60 & 26,7 & 93 & 97 & 33,3 & 18,2 & 97,5 & 98,2 & 8,6 & 9,2 & 0,43 & 0,75 \\
\hline $\begin{array}{l}\text { Harrington+ } \\
2004^{34}\end{array}$ & 628 & 95 & 88,9 & 80 & 74,5 & 38,8 & 16,3 & 99,2 & 99,2 & 4,9 & 3,4 & 0,06 & 0,15 \\
\hline $\begin{array}{l}\text { Axt-Fliedner } \\
2005^{35}\end{array}$ & 52 & 50 & $n / d$ & 75 & $n / d$ & 14 & $n / d$ & 95 & $n / d$ & 2 & $n / d$ & 0,66 & $n / d$ \\
\hline $\begin{array}{l}\text { Papageorghiou } \\
2005^{36}\end{array}$ & 16806 & 45 & $n / d$ & 90 & $\mathrm{n} / \mathrm{d}$ & 9,1 & $n / d$ & 98 & $n / d$ & 5,07 & $n / d$ & 0,6 & $n / d$ \\
\hline Ay $2005^{32}$ & 178 & 78 & $n / d$ & 86,6 & $n / d$ & 33 & $n / d$ & 97,9 & $n / d$ & 5,8 & $n / d$ & 0,25 & $n / d$ \\
\hline Yu $2005^{37}$ & 32157 & 56 & 82 & 90 & 90 & 9,8 & 3,5 & 99 & 99,9 & 5,6 & 8,2 & 0,48 & 0,2 \\
\hline Parra $2005^{22}$ & 743 & 48 & 85 & 96 & 95 & 24,6 & 18 & 98,5 & 99,8 & 11,5 & 18,8 & 0,54 & 0,15 \\
\hline Yu $2008^{38}$ & 30639 & 22 & 77 & 95 & 95 & 8,3 & $n / d$ & 98,3 & $n / d$ & 4,4 & 15,4 & 0,92 & 0,24 \\
\hline $\begin{array}{l}\text { Onwudiwe } \\
2008^{33}\end{array}$ & 3347 & 56 & 100 & 90 & 90 & 17,5 & 6,4 & 98,5 & 100 & 5,6 & 10 & 0,48 & 0 \\
\hline
\end{tabular}

+ Valores obtenidos para población embarazada con alto riesgo de PE. El resto de los valores fueron obtenidos en población general de embarazadas. $\mathrm{n} / \mathrm{d}=$ no disponible. S: sensibilidad. E: especificidad. VPP: valor predictivo positivo. VPN: valor predictivo negativo. LR: likelihood ratio. PE: preeclampsia. PE pr: Preeclampsia precoz.

\section{DISCUSIÓN}

La PE, como se entiende actualmente, es una enfermedad que se puede subdividir en tardía (habitualmente sin RCF, moderada, sin repercusión materna ni fetal) y precoz (antes de las 34 semanas, habitualmente acompañada de RCF, severa, con repercusiones maternas y/o fetales), que se asocia a defectos de la invasión trofoblástica en los vasos uterinos y que se manifiesta, frecuentemente, como DAUt alterado (7).

Analizamos un total de 38 trabajos (incluyendo 32 originales, 5 revisiones y 1 meta-análisis) que evalúan la utilidad del DAUt alterado como método de tamizaje de PE y la utilidad del AAS para prevenir la aparición de PE.

El análisis de la literatura disponible nos permite interpretar que el DAUt en segundo trimestre asociado a los factores de riesgo maternos (embarazos de alto riesgo), ofrece la oportunidad de detectar la PE precoz con la mejor S ( 80\%), E ( 95\%) y VPN $(\sim 99 \%)$, con VPP $(\sim 18 \%)$, LR+ $(\sim 15)$ y LR- $(\sim 0,2)$ que distan de entregarnos un pronóstico aceptable (ver ejemplo, Figura 1). Todos los resultados reportados para predicción de PE en globo (o tardía) y otros resultados perinatales adversos (RCIU, óbito fetal, desprendimiento de placenta) son aún me- nos exactos, por lo que no recomendamos el DAUt como método de tamizaje en población general.

La administración de AAS en bajas dosis se ha mostrado efectiva en la prevención de PE en población de riesgo, pero con disminuciones marginales $(\sim 17 \%)$ en su incidencia y sin disminuir la morbimortalidad materna ni perinatal (39). Se necesita tratar a 72 pacientes para prevenir un caso de PE. Los estudios que han utilizado el AAS en bajas dosis en población de alto riesgo tamizada con DAUt tienen resultados no concluyentes y el meta-análisis disponible, a nuestro modo de análisis, adolece de falencias metodológicas, por lo que se necesitan nuevos estudios aleatorizados y controlados, que utilicen AAS o placebo si el DAUt en primer o segundo trimestre se encuentra alterado, con diseños adecuados para dilucidar esta disyuntiva.

Asimismo, se necesita más evidencia para avalar el uso de marcadores séricos, idealmente, con análisis costo-beneficio, considerando que nuestro sistema de salud cuenta con recursos económicos limitados. Actualmente hay dos estudios registrados en el ICTRP que evalúan la utilidad de AAS en bajas dosis en pacientes de alto riesgo seleccionadas por historia materna y DAUt alterado, y otro que evalúa la utilidad de heparina no fraccionada en el mismo tipo de pacientes. Si se comprobara la 
existencia de una intervención eficaz en prevenir el desarrollo de PE precoz (severa), el DAUt se justificaría plenamente en población seleccionada (de alto riesgo) como método de tamizaje.

\section{CONCLUSIÓN}

El DAUt alterado es un método útil para el tamizaje de PE precoz en pacientes embarazadas seleccionadas por factores de riesgo, pero se necesitan más estudios evaluando la utilidad del AAS como profilaxis de PE en dicha población.

\section{REFERENCIAS}

1. Sibai B, Dekker G, Kupferminc M. Preeclampsia. Lancet 2005;365:785-99.

2. Ray JG, Vermeulen MJ, Schull MJ, Redelmeier DA. Cardiovascular health after maternal placental syndromes (CHAMPS): population-based retrospective cohort study. Lancet 2005;366:1797-1803.

3. Barker DJ. Adult consequences of fetal growth restriction. Clin Obstet Gynecol 2006;49: 270-83.

4. Gluckman PD, Hanson MA, Cooper C, Thornburg $\mathrm{KL}$. Effect of in utero and early-life conditions on adult health and disease. N Engl J Med 2008;359:61-73.

5. Norwitz E, Lockwood C, Barss V. Prediction of preeclampsia. UpToDate 2010;18.2.

6. Levine, RJ, Lindheimer, MD. First-trimester prediction of early preeclampsia: a possibility at last! Hypertension 2009;53:747-8.

7. Dekker GA, Sibai BM. Etiology and pathogenesis of preeclampsia: current concepts. Am J Obstet Gynecol 1998;179:1359-75.

8. Zhong $\mathrm{Y}$, Tuuli $\mathrm{M}$, Odibo AO. First-trimester assessment of placenta function and the prediction of preeclampsia and intrauterine growth restriction. Prenat Diagn $2010 ; 30: 293-308$.

9. Yu CK, Smith GC, Papageorghiou AT, Cacho AM, Nicolaides $\mathrm{KH}$; Fetal Medicine Foundation Second Trimester Screening Group. An integrated model for the prediction of preeclampsia using maternal factors and uterine artery Doppler velocimetry in unselected lowrisk women. Am J Obstet Gynecol 2005;193:429-36.

10. Duckitt K, Harrington D. Risk factors for pre-eclampsia at antenatal booking: a systematic review of controlled studies. BMJ 2005;330:565.

11. Wilkström AK, Stephansson O, Cnattingius S. Tobacco use during pregnancy and pre-eclampsia risk: effects of cigarette smoking and snuff. Hypertension 2010; 55:1254-9.

12. Nicolaides KH, Bindra R, Turan OM, Chefetz I, Sammar M, Meiri H, Tal J, Cuckle HS. A novel approach to first-trimester screening for early pre-eclampsia combining serum PP-13 and Doppler ultrasound. UItrasound Obstet Gynecol 2006;27:13-7.

13. Spencer K, Yu CK, Savvidou M, Papageorghiou AT, Nicolaides $\mathrm{KH}$. Prediction of pre-eclampsia by uterine artery Doppler ultrasonography and maternal serum pregnancy-associated plasma protein-A, free beta-hu- man chorionic gonadotropin, activin $\mathrm{A}$ and inhibin $\mathrm{A}$ at $22+0$ to $24+6$ weeks' gestation. Ultrasound Obstet Gynecol 2006;27:658-63.

14. Spencer K, Cowans NJ, Chefetz I, Tal J, Meiri H. First-trimester maternal serum PP-13, PAPP-A and second-trimester uterine artery Doppler pulsatility index as markers of pre-eclampsia. Ultrasound Obstet Gynecol 2007;29:128-34.

15. Brown MA, Lindheimer MD, deSweit M, Van Assche A, Moutquin J. The classification and diagnosis of the hypertensive disorders of pregnancy: statement from the international society for the study of hypertension in pregnancy (ISSHP). Hypertens Pregnancy 2001;20:9-14.

16. Carvajal J. Guía para el análisis crítico de publicaciones científicas. Rev Chil Obstet Ginecol 2004;69:67-72.

17. Vera C, Letelier L, Carvajal J. Guía para el análisis crítico de estudios que evalúan exámenes diagnósticos. Rev Chil Obstet Ginecol 2005;70:196-202.

18. Campbell S, Diaz-Recasens J, Griffin DR, CohenOverbeek TE, Pearce JM, Willson K, Teague MJ. New Doppler technique for assessing uteroplacental blood flow. Lancet 1983;26:675-7.

19. Papageorghiou AT, Yu CK, Nicolaides KH. The role of uterine artery Doppler in predicting adverse pregnancy outcome. Best Pract Res Clin Obstet Gynaecol 2004;18:383-96.

20. von Dadelszen P, Magee LA, Roberts JM. Subclassification of pre-eclampsia. Hypertens Pregnancy 2003;22:143-8.

21. Martin AM, Bindra R, Curcio I, Cicero S, Nicolaides $\mathrm{KH}$. Screening for pre-eclampsia and fetal growth restriction by uterine artery Doppler at 11-14 weeks of gestation. Ultrasound Obstet Gynecol 2001;18:583-6.

22. Parra M, Rodrigo R, Barja $P$, Bosco $C$, Fernández V, Muñoz H, Soto-Chacón E. Screening test for preeclampsia through assessment of uteroplacental blood flow and biochemical markers of oxidative stress and endothelial dysfunction. Am J Obstet Gynecol 2005;193:1486-91

23. Gómez O, Martínez JM, Figueras F, Del Río M, Borobio V, Puerto B, Coll O, Cararach V, Vanrell JA. Uterine artery Doppler at 11-14 weeks of gestation to screen for hypertensive disorders and associated complications in an unselected population. Ultrasound Obstet Gynecol 2005;26:490-4.

24. Pilalis A, Souka AP, Antsaklis $P$, Basayiannis K, Benardis $P$, Haidopoulos D, Papantoniou N, Mesogitis S, Antsaklis A. Screening for pre-eclampsia and small for gestational age fetuses at the 11-14 weeks scan by uterine artery Dopplers. Acta Obstet Gynecol Scand 2007;86:530-4.

25. Audibert F, Boucoiran I, An N, Aleksandrov N, Delvin E, Bujold E, Rey E. Screening for preeclampsia using first-trimester serum markers and uterine artery Doppler in nulliparous women. Am J Obstet Gynecol 2010;203:383.e1-8.

26. Plasencia W, Maiz N, Bonino S, Kaihura C, Nicolaides $\mathrm{KH}$. Uterine artery Doppler at $11+0$ to $13+6$ weeks in the prediction of pre-eclampsia. Ultrasound Obstet Gynecol 2007;30:742-9.

27. Poon LC, Staboulidou I, Maiz N, Plasencia W, Ni- 
colaides $\mathrm{KH}$. Hypertensive disorders in pregnancy: screening by uterine artery Doppler at 11-13 weeks. Ultrasound Obstet Gynecol 2009;34:142-8.

28. Poon LC, Karagiannis G, Leal A, Romero XC, Nicolaides $\mathrm{KH}$. Hypertensive disorders in pregnancy: screening by uterine artery Doppler imaging and blood pressure at 11-13 weeks. Ultrasound Obstet Gynecol 2009;34:497-502.

29. Poon LC, Stratieva V, Piras S, Piri S, Nicolaides $\mathrm{KH}$. Hypertensive disorders in pregnancy: combined screening by uterine artery Doppler, blood pressure and serum PAPP-A at 11-13 weeks. Prenat Diagn 2010;30:216-23.

30. Papageorghiou AT, Yu CK, Bindra R, Pandis G, Nicolaides $\mathrm{KH}$; Fetal Medicine Foundation Second Trimester Screening Group. Multicenter screening for pre-eclampsia and fetal growth restriction by transvaginal uterine artery Doppler at 23 weeks of gestation. Ultrasound Obstet Gynecol 2001;18:441-9.

31. Aquilina J, Thompson O, Thilaganathan B, Harrington K. Improved early prediction of pre-eclampsia by combining second-trimester maternal serum inhibin-A and uterine artery Doppler. Ultrasound Obstet Gynecol 2001;17:477-84.

32. Ay E, Kavak ZN, Elter K, Gokaslan H, Pekin T. Screening for pre-eclampsia by using maternal serum inhibin $A$, activin $A$, human chorionic gonadotropin, unconjugated estriol, and alpha-fetoprotein levels and uterine artery Doppler in the second trimester of pregnancy. Aust N Z J Obstet Gynaecol 2005;45:283-8.

33. Onwudiwe N, Yu CK, Poon LC, Spiliopoulos I, Nicolaides $\mathrm{KH}$. Prediction of pre-eclampsia by a combination of maternal history, uterine artery Doppler and mean arterial pressure. Ultrasound Obstet Gynecol 2008;32:877-83.

34. Harrington K, Fayyad A, Thakur V, Aquilina J. The value of uterine artery Doppler in the prediction of uteroplacental complications in multiparous women. Ultrasound Obstet Gynecol 2004;23:50-5.

35. Axt-Fliedner R, Schwarze A, Nelles I, Altgassen C, Friedrich M, Schmidt W, Diedrich K. The value of uterine artery Doppler ultrasound in the prediction of severe complications in a risk population. Arch Gynecol Obstet 2005;271:53-8.

36. Papageorghiou AT, Yu CK, Erasmus IE, Cuckle HS, Nicolaides $\mathrm{KH}$. Assessment of risk for the development of pre-eclampsia by maternal characteristics and uterine artery Doppler. BJOG 2005;112:703-9.

37. Yu CK, Smith GC, Papageorghiou AT, Cacho AM, Nicolaides $\mathrm{KH}$; Fetal Medicine Foundation Second Trimester Screening Group. An integrated model for the prediction of preeclampsia using maternal factors and uterine artery Doppler velocimetry in unselected lowrisk women. Am J Obstet Gynecol 2005;193:429-36.

38. Yu CK, Khouri O, Onwudiwe N, Spiliopoulos Y, Nicolaides $\mathrm{KH}$; Fetal Medicine Foundation Second-Trimester Screening Group. Prediction of pre-eclampsia by uterine artery Doppler imaging: relationship to gestational age at delivery and small-for-gestational age. Ultrasound Obstet Gynecol 2008;31:310-3.

39. Duley L, Henderson-Smart DJ, Meher S, King JF. Antiplatelet agents for preventing pre-eclampsia and its complications. Cochrane Database Syst Rev. 2007;(2):CD004659.

40. Goffinet F, Aboulker D, Paris-Llado J, Bucourt M, Uzan M, Papiernik E, Bréart G. Screening with a uterine Doppler in low risk pregnant women followed by low dose aspirin in women with abnormal results: a multicenter randomised controlled trial. BJOG 2001;108:510-8.

41. Subtil D, Goeusse P, Houfflin-Debarge V, Puech F, Lequien P, Breart G, Uzan S, Quandalle F, Delcourt YM, Malek YM; Essai Régional Aspirine Mère-Enfant (ERASME) Collaborative Group. Randomised comparison of uterine artery Doppler and aspirin (100 $\mathrm{mg}$ ) with placebo in nulliparous women: the Essai Régional Aspirine Mère-Enfant study (Part 2). BJOG 2003;110:485-91.

42. Yu CK, Papageorghiou AT, Parra M, Palma Dias R, Nicolaides $\mathrm{KH}$; Fetal Medicine Foundation Second Trimester Screening Group. Randomized controlled trial using low-dose aspirin in the prevention of preeclampsia in women with abnormal uterine artery Doppler at 23 weeks' gestation. Ultrasound Obstet Gynecol 2003;22:233-9.

43. Ebrashy A, Ibrahim M, Marzook A, Yousef D. Usefulness of aspirin therapy in high-risk pregnant women with abnormal uterine artery Doppler ultrasound at 1416 weeks pregnancy: randomized controlled clinical trial. Croat Med J 2005;46:826-31.

44. Fox C, Khan KS, Coomarasamy A. How to interpret randomised trials of test-treatment combinations: a critical evaluation of research on uterine Doppler test to predict, and aspirin to prevent, pre-eclampsia. BJOG 2010;117:801-8. 